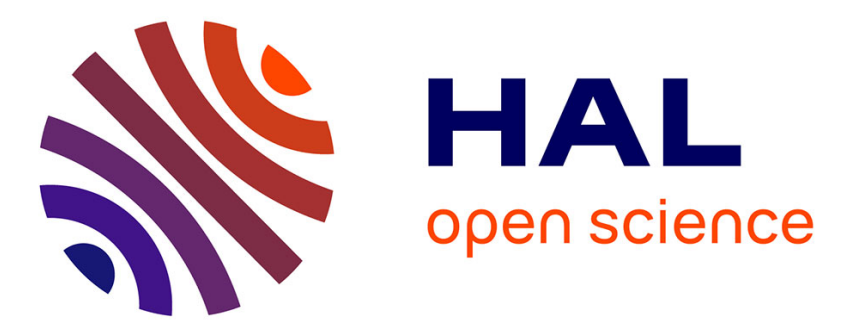

\title{
Response of a Noncohesive Packing of Grains to a Localized Force: Deviation From Continuum Elasticity.
} Jean Rajchenbach, Amar El Hadji Bouya, Didier Clamond, Nathalie Fraysse

\section{To cite this version:}

Jean Rajchenbach, Amar El Hadji Bouya, Didier Clamond, Nathalie Fraysse. Response of a Noncohesive Packing of Grains to a Localized Force: Deviation From Continuum Elasticity.. Physical Review E: Statistical, Nonlinear, and Soft Matter Physics, 2011, 83 (2), pp.021304. 10.1103/PhysRevE.83.021304 . hal-00567126

\section{HAL Id: hal-00567126 https://hal.science/hal-00567126}

Submitted on 18 Feb 2011

HAL is a multi-disciplinary open access archive for the deposit and dissemination of scientific research documents, whether they are published or not. The documents may come from teaching and research institutions in France or abroad, or from public or private research centers.
L'archive ouverte pluridisciplinaire HAL, est destinée au dépôt et à la diffusion de documents scientifiques de niveau recherche, publiés ou non, émanant des établissements d'enseignement et de recherche français ou étrangers, des laboratoires publics ou privés. 


\title{
Response of a Noncohesive Packing of Grains to a Localized Force: Deviation From Continuum Elasticity.
}

\author{
El Hadji Bouya Amar ${ }^{1}$, D. Clamond ${ }^{2}$, N. Fraysse ${ }^{1}$, J. Rajchenbach ${ }^{1}$ \\ 1 Laboratoire de Physique de la Matière Condensée (CNRS-UMR 6622), \\ Université de Nice Sophia Antipolis, \\ Parc Valrose, F-06108 Nice Cedex 2, France \\ 2 Laboratoire Jean-Alexandre Dieudonné (CNRS UMR 6621), \\ Université de Nice Sophia Antipolis, \\ Parc Valrose, F-06108 Nice Cedex 2, France
}

\begin{abstract}
In order to characterize the mechanical behavior of grain piles, we investigate the response of a non-cohesive two-dimensional packing of cylinders submitted to a localized force. By means of image processing, we obtain an accurate measurement of the individual grain displacements in the reversible regime of deformation. The measured displacement field deviates unambiguously from the predictions of linear elasticity and of other theoretical descriptions commonly used to model the behavior of cohesionless soils in civil engineering or in soil mechanics. Surprisingly, the analysis of the deformation field reveals a tendency to localization in the reversible regime.
\end{abstract}

PACS numbers: 45.70-n, 45.70-cc, $83.10 \mathrm{Gr}, 83.80 \mathrm{Fg}$

Published in Phys. Rev. E 83, 021304 (2011)

Non-cohesiveness is an essential feature distinguishing grain packings from continuous media, because the former cannot resist tensile stresses. In such packings, grains interact via elastic contact forces and by solid friction. The relationship between contact forces and individual grain deformations is essentially nonlinear, as exemplified by the Hertz-Mindlin law valid for spherical grains [1-3]. It is worth noting that the number of contact forces between neighboring grains is generally larger than the number of equilibrium mechanical equations: this leads to multi-valued equilibrium solutions [4-6]. These equilibrium equations have to be supplemented by a set of inequalities, stating that the contact forces exist in the Coulomb cone. This set of inequalities precludes any variational formulation to determine the contact forces, unlike the case of elasticity [7]. Moreover, for a loose packing, new contacts are created as the confining pressure is increased. All these considerations cast doubt on the applicability of the linear elasticity theory for modeling the behavior of dry grain packings. Nevertheless, according to classical civil engineering textbooks [8], grain piles or soils submitted to a purely compressive external load are considered to obey linear elasticity below the Mohr-Coulomb plastic yield criterion. In the elastic equilibrium state, the stress field obeys the Beltrami biharmonic equation, which is of elliptic nature. This description implies therefore the uniqueness of the solution for the internal stress field and for the deformation field, for a given set of boundary conditions. The existence of multiple equilibrium states for the ensemble of contact forces in the microscopic description calls into question the uniqueness expected from the elastic modeling in the continuous limit. Note moreover that for a grain pile there is a memory effect, that is, a dependence of the internal state of stress on the preparation process.
This memory effect was first recognized by Darwin more than one century ago [9], and then reassessed by Geng et al. [10]. The memory effect implies the existence of multiple equilibrium states for identical boundary conditions, which obviously opposes the elastic description.

In order to gain insight into the nature of the stress equilibrium equations, we performed a point-punch test onto a two-dimensional packing. The point-punch test represents the Green function of the mechanical response for the discrete medium, provided it is linear (note that this last assumption is also questionable [11]). The sample studied is prepared by cutting a 10 -mm-thick elastomeric plate into grains that are identical cylinders $(8$ $\mathrm{mm}$ diameter, $10 \mathrm{~mm}$ long). The two-dimensional collection of grains is then packed into contact according to a triangular compact lattice (i.e., centered hexagonal) and bounded by a rigid hexagonal metal frame. The frame ensures zero normal displacement boundary conditions. The Young's modulus and the Poisson ratio of the polyurethane elastomer are respectively $E=4 \cdot 10^{6} \mathrm{~Pa}$ and $\nu=0.46$. The punch consists of a steel blade of 10 $\mathrm{mm}$ long, $3 \mathrm{~mm}$ thick and a Young's modulus of $2 \cdot 10^{11}$ $\mathrm{Pa}$. The elastomeric material has been chosen because the small Young's modulus allows a high relative precision in the measurement of the deformations induced by a gentle point load. Note that the 2D triangular packing (in-plane strain) can be considered as isotropic from the viewpoint of linear elasticity [12].

Previous experimental studies probing the response of a granular piling submitted to a point load have led to controversial analyses. Using a photo-elastic visualization method and a piling consisting of square tiles, Da Silva and Rajchenbach [13] concluded that their observations cannot be interpreted in the framework of linear elastic modeling, and rather supported a hierarchical 
process for the stress transmission, consistently with the models of Harr [14] and of Coppersmith et al. [15]. Indeed, in two dimensions, the elastic response of a semiinfinite medium to a point normal force can be described as follows [16]. If the origin (of polar coordinates $r, \theta$ ) is taken as the point of application of the load $P$ (defined as a force per unit of length), the stress is everywhere radial, and its magnitude is given by $\sigma(r, \theta)=(2 P / \pi r) \cos \theta$. Hence the contours of constant stress magnitude are a set of circles passing through the point of application of the force. This result holds for a semi-infinite elastic medium and the iso-stress contours are slightly modified in the presence of rigid boundary conditions, as discussed below. By means of the same photo-elastic method, but using pentagonal grains, Geng et al. [17] observed a wedgeshaped brightened domain (with a wedge angle close to $60^{\circ}$ ), which does not correspond to the circular iso-stress contours expected from isotropic linear elasticity. Another interesting study is that of Goldenberg et al. [18] who probed the response of a $2 \mathrm{D}$ triangular packing by means of numerical simulations. Unilateral (i.e., noncohesive) normal contact forces were modeled by one-sided linear springs, and the tangential friction between grains was disregarded. In the limit of large systems, this model was shown to obey linear elasticity, which was consistent with the fact that the elastic solution as a response to a compressive load involves no tensile stress. In our experimental procedure, a sequence of pictures is taken as the external load is increased. Then successive pictures are processed in order to access the displacement field. For the sake of accuracy, the bulk of the grains has been seeded with fine tracer particles $(0.2 \mathrm{~mm}$ in diameter) which allow a precise tracking of local displacements. The measurement accuracy is of the order of 2 pixels, which corresponds to $2 / 100$ particle diameter. The experimental load cell is sketched in Fig. 1.

Figure 2 depicts a 3D representation of the local displacement amplitude as a function of the position $(x, y)$, resulting from a punch indentation of $2 \mathrm{~mm}$. Note that the observed fluctuations should not be attributed to limitations on the measurement accuracy, but to the inhomogeneities in the displacements on the scale of one grain. Indeed, the tracers located in the vicinity of contacts experience a displacement much larger than that undergone by the tracers located near the center of the same grain. It is of interest to compare the displacement field to that obtained in the case of a continuous elastic material. With that aim, we submitted a continuous plate of the same elastomeric material, of identical thickness $(10 \mathrm{~mm})$, positioned in the same hexagonal frame to the same punching test. In Fig. 3, we show the displacements plotted as a function of the distance from the punch, measured along the direction of loading $O y$, both for the discrete packing and for the continuous plate. Moreover, in order to compare the actual measured response and the theoretical elastic predictions, we show in

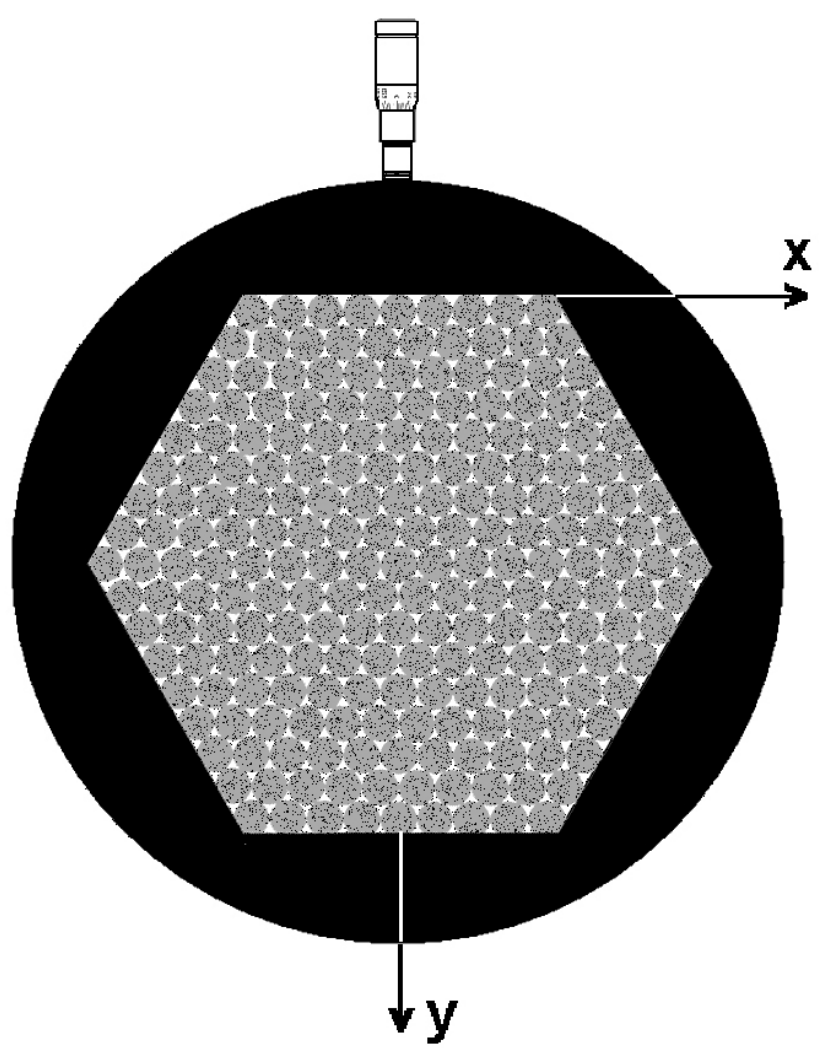

FIG. 1: Sketch of the load cell. The collection of cylinders is packed into a triangular (centered hexagonal) compact lattice, and confined into a rigid hexagonal frame. Cylinders are 8 $\mathrm{mm}$ in diameter. Grains are seeded with tracer particles. The displacements of the tracers are tracked as the punch is progressively moved inwards.

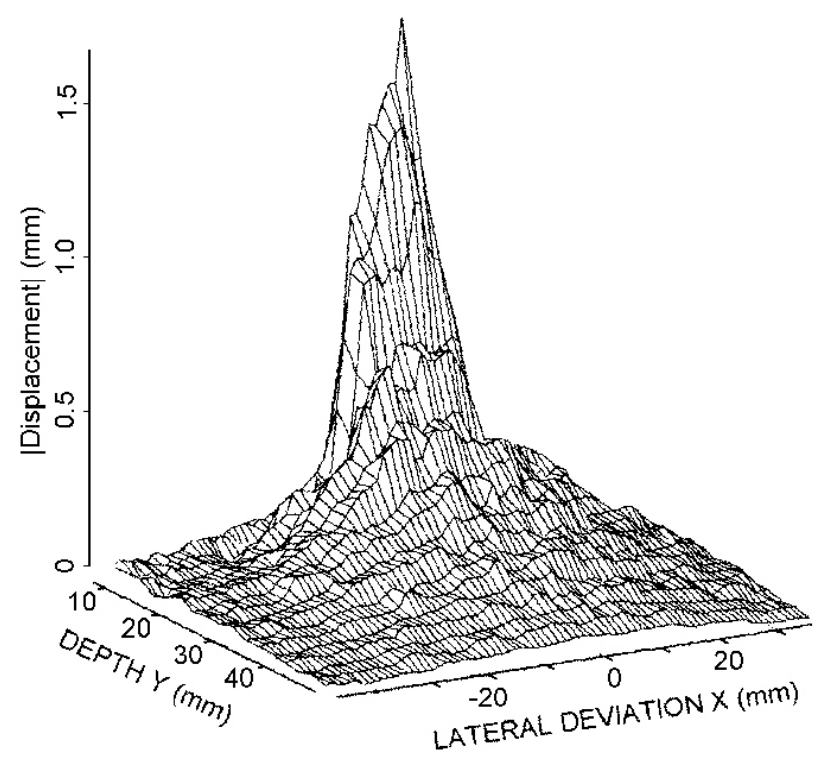

FIG. 2: 3D representation of the local displacement field obtained in the $2 \mathrm{D}$ packing of cylinders for a punch indentation of $2 \mathrm{~mm}$. 


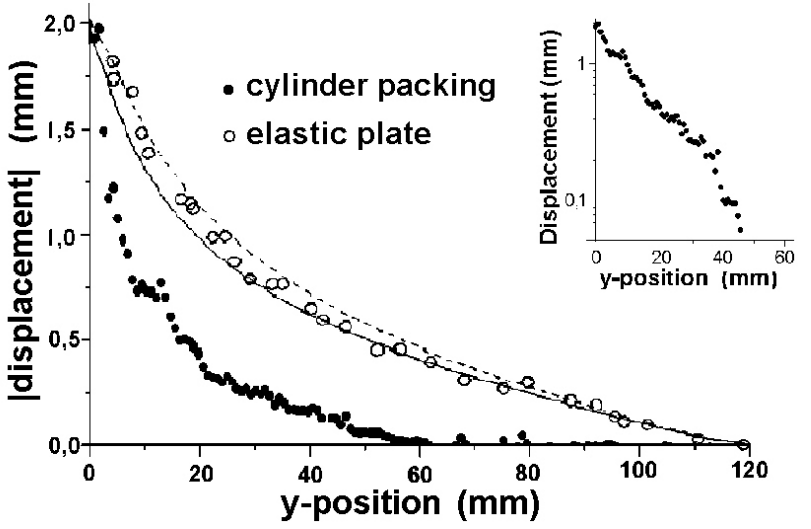

FIG. 3: Displacement amplitude, measured along the load axis $O y$ ( $\bullet$ discrete piling, o continuous plate) and elastic predictions for various Poisson moduli in a plane strain modeling (continuous line: $\nu=-0.9$, dashed line $\nu=0.5$ ). In inset, semi-log plot of the displacement amplitude as a function of $y$.

the same figure the displacement curves obtained by solving analytically the Navier-Lamé equation (with Poisson ratios $\nu=-0.9$ and $\nu=0.5$ ) with the same boundary conditions, and in the planar strain configuration. Note that a variation in the Young's modulus would be ineffective on displacements along the punching axis, since the present boundaries impose conditions on displacements, not on stresses. The experimental points obtained for the continuous plate compare well with the theoretical elastic description, and thus clearly indicates an elastic response of the polymeric material. On the other hand, the displacement field corresponding to the 2D discrete packing deviates unambiguously from the predictions of the linear elasticity of continuous media. Instead, it follows roughly an exponential-like decay with the distance along the direction of loading (see inset, Fig. 3). The exponential-like decay along $O y$ suggests a localization process.

To sharpen our comparison of the mechanical response of a $2 \mathrm{D}$ granular packing to a punch, with that of a continuous elastic medium, it is interesting to examine the displacement field in locations off the axis of loading. In Fig. 4, we show the map of the displacement amplitude as a function of the position $(x, y)$, coded in gray levels. Apart from the fluctuations originating in the position of the tracers, according to their distance relative to grain centers or to contacts (as indicated above), it is clear that the iso-displacement contours resemble closely a family of ellipses.

From an assumed exponential-like decay of the displacements according to the punching axis direction, and of the elliptic-like shape of the iso-displacement contours in the $(x, y)$ plane, elementary geometrical considerations

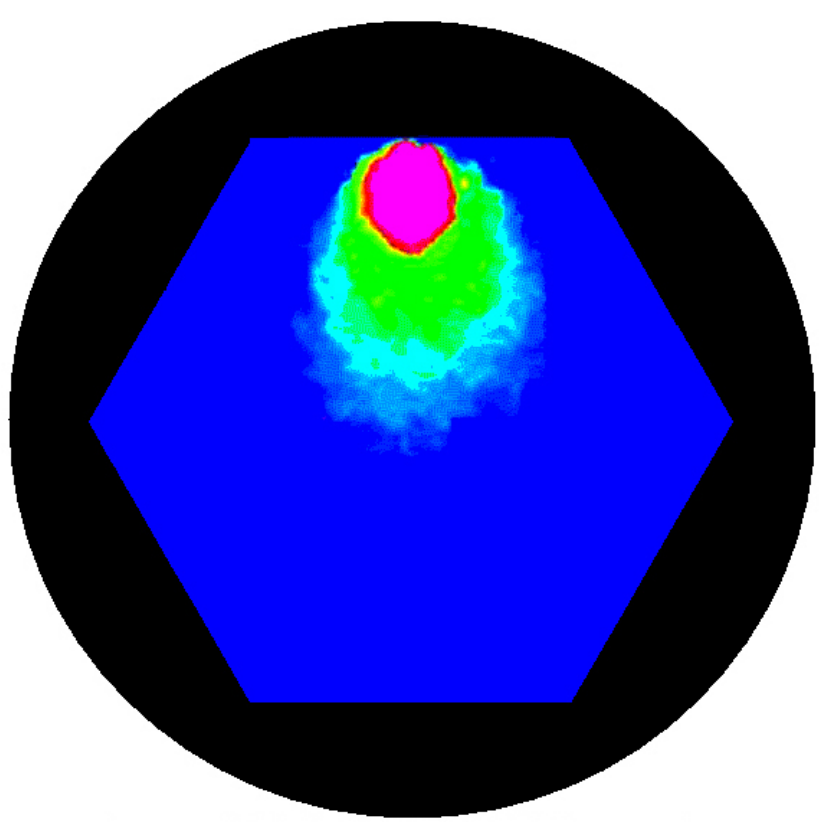

FIG. 4: Same data as Fig. 2. Displacement amplitude as a function of the position $(x, y)$, coded in color-levels. The contours corresponding to displacements of equal amplitude are approximately elliptic.

lead to the following form for the displacement magnitude in the discrete medium:

$$
\text { | displacement } \mid \propto \exp (-y / a) \exp \left(-x^{2} / b y\right)
$$

where the lengths $a$ and $b$ are here of comparable magnitude, typically 3 to 4 grain diameters. To confirm the validity of relation (1), we have plotted in Fig. 5 the displacement amplitude as a function of the transverse position $x$, for various ordinates $y$. It is clear that the set of experimental data can reasonably be fitted by a family of Gaussian curves, with standard deviation varying as $\sqrt{y}$. Note that the data obtained by Da Silva et al. [13] can be accounted for by the same fitting function (1), with $a \simeq 4$ and $b \simeq 0.5$ grain diameters. The Gaussian widening along $O x$ is reminiscent of the diffusive models, previously proposed by Harr [14] and by Coppersmith et al. [15]. However, note that the latter models predict a $1 / \sqrt{y}$ decay along the direction of loading, rather than the exponential-like one, as observed. At this point, it is worth addressing the issue of sample size, i.e., the cell size compared to the grain diameter. The packing comprises about 220 grains. As shown above, the localization length is typically 4 grain diameters, and the tracer displacements attain a zero value (within the experimental accuracy) at positions far from the cell boundaries. We conclude that using larger cells (with rigid boundaries imposing a zero normal displacement) would not lead to any significant change in either the localization phenomena or the reported localization 


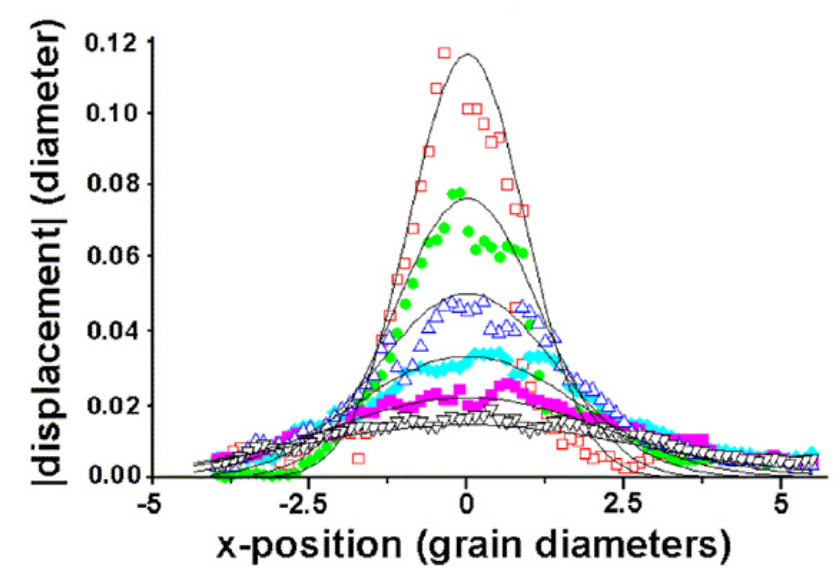

FIG. 5: Displacement amplitude as a function of the deviation $x$ to the axis of punching, plotted for various ordinates y ( $\square$, $y=3.1 \mathrm{~mm}),(\bullet, y=10.0 \mathrm{~mm}),(\triangle, y=17.0 \mathrm{~mm}),(\diamond$, $y=21.0 \mathrm{~mm}),(\boldsymbol{\square}, y=30.0 \mathrm{~mm}),(\nabla, y=39.0 \mathrm{~mm})$. Continuous lines correspond to Gaussian fits according to Eq. (1) (see text). The lengths $a$ and $b$ appearing in Eq. (1) are of comparable magnitude, typically 3 or 4 grain diameters.

lengths.

As emphasized above, our data are incompatible with the predictions of linear elasticity commonly used in civil engineering to model the behavior of noncohesive soils below plastic yielding. It is worth noting that our experimental results also contradict some alternative theoretical approaches aimed at modeling the equilibrium behavior of granular assemblies, such as micropolar elasticity [19], micropolar elasto-plasticity [20], hypoplastic theory [21], and the hyperbolic model of Bouchaud et al. [22].

Indeed, micropolar elasticity does not predict any localization effects in the response to a point load. Taking into account micropolar effects and couple stresses brings only negligible corrections (acting as $1 / r^{3}$ with the distance to the punch) to the stress field obtained within the frame of continuum elasticity (which decreases as $1 / r$ ) (e.g. see [23]). On the other hand, micropolar elasto-plasticity predicts strain localization effects and the formation of shear bands at the plastic yielding. Thus, the localization process is predicted as irreversible. Irreversibility is of course also obtained within the frame of hypoplasticity. Concerning the hyperbolic model of Bouchaud et al. [22], the observed response is fully incompatible with the picture of internal stresses confined in two "rays" starting from the loading point.

In summary, we have performed accurate measurements of the displacement field in a two-dimensional packing of elastic grains, as a response to a point load in the reversible regime of deformation. The coarsegrained displacement field deviates unambiguously from the predictions of continuum linear elasticity. We have found that the deformation amplitude, measured along the punching axis $O y$, follows an exponential-like decay as a function of the distance $y$ to the punch, which indicates a localization process. On the other hand, the isodisplacement contours resemble a family of ellipses, and the transverse width of the strained region varies approximately as $\sqrt{y}$. We emphasize that, although each individual grain behaves elastically, the collective response is reversible, so that the coarse-grained strain field does not map onto linear elasticity predictions. Moreover, current alternative models devised to overcome some known limitations of the standard elasto-plastic model seem to fail in accounting for our data.

[1] H. Hertz, J. Reine Angew. Math. 92, 156 (1881).

[2] R. D. Mindlin, J. Appl. Mech. (ASME) 16, 259 (1949).

[3] The elastic displacement $\delta$ of two equal spheres submitted to a compressive normal force $F$ reads as $\delta=$ $\left[9\left(1-\nu^{2}\right)^{2} / 16 E^{2} R\right]^{1 / 3} F^{2 / 3}$ (see Ref. [1]). For two cylinders of length $l$ maintained in contact along a generatrix, the displacement is $\delta=\left[2 F\left(1-\nu^{2}\right) / \pi E l\right][\ln (4 \pi E R l /(1-$ $\left.\left.\nu^{2}\right) F\right)-1$ in the plane strain approximation (see K.L. Johnson, Contact mechanics, Cambridge University Press, Cambridge, U.K., 1985).

[4] S. Alexander, Phys. Reports 296, 65 (1998).

[5] J.J. Moreau, Indétermination Liée au Frottement Sec dans le Calcul des Granulats, in Actes du 6me Colloque en Calcul des Structures, M. Pottier-Ferry et al. Eds, Vol. 3, page 465, Teknea, Toulouse 2003.

[6] T. Unger, J. Kertész and D. Wolf, Phys. Rev. Lett. 94, 178001 (2005).

[7] Note that there is attempts to describe the Coulombian friction within a bipotential formalism. This description succeeds in accounting for the plurality of solutions (see M. Buliga, G. De Saxcé and C. Vallée, Journal of Convex Analysis 17, 081 (2010)).

[8] K. Terzaghi, Theoretical Soil Mechanics (John Wiley \& Son, New York, 1943).

[9] G.H. Darwin, Minutes of the Proc. Inst. Civ. Eng. 71, 350 (1883).

[10] J. Geng, E. Longhi, R.P. Behringer, and D. Howell, Phys. Rev. E. 64, R060301 (2001).

[11] J. J. Moreau, Oral communication, G.D.R. MIDI Meeting, Carry le Rouet (2004, unpublished).

[12] M. H. Sadd, Qingli Dai, Mechanics of Materials 37, 641 (2005).

[13] M. Da Silva, J. Rajchenbach, Nature 406, 708 (2000).

[14] M.E. Harr, Foundations of Theoretical Soil Mechanics (McGraw-Hill, 1966).

[15] S.N. Coppersmith, C.H. Liu, S. Majumdar, O. Narayan, T.A. Witten, Phys. Rev. E 53, 4673 (1996).

[16] M. Flamant, Comptes Rendus Hebdomadaires des Séances de l'Académie des Sciences (Paris) 114, 1465 (1892).

[17] Junfei Geng, D. Howell, E. Longhi, R.P. Behringer, G. 
Reydellet, L. Vanel and E. Clément, Phys. Rev. Lett. 87, 035506 (2001).

[18] C. Goldenberg and I. Goldhirsch, Phys. Rev. Lett. 89 , 084302 (2002).

[19] A. C. Eringen, in Fracture II, edited by H. Leibovitz (Academic, New York, 1968).

[20] H.-B. Mulhaus \& I. Vardoulakis, Géotechnique 37, 271 (1987).
[21] D. Kolymbas, Introduction to Hypoplasticity, Balkema, Rotterdam (2000).

[22] J. P. Bouchaud, M. E. Cates, \& P. Claudin, J. Phys. I 5, 639 (1995)

[23] W. Nowacki, in Prípevsky k teórii stavebných konstrukcií, p. 76, Veda, vydavatelstvo Slovenskej akadémie vied Ed., (Bratislava, 1974). 